\title{
Reprises et modifications des explications d'un conte moral par des enfants de cinq ans
}

\author{
Marie Carcassonne \\ IRISES-CERSO (UMR 7170) \\ Mireille Froment \\ Modèles, Dynamiques, Corpus (MoDyCo) \\ Nathalie Salagnac \\ THEODILE EA 1764 \\ Université Lille3
}

\section{- 1 Une conception dialogique du récit}

Plus qu'il ne l'intériorise, l'enfant «reprend-modifie» (François, 1993) la culture de l'adulte, en l'interprétant d'une façon qui lui est propre : un processus dialogique de circulation des significations est à l'œuvre (Bakhtine, 1977, Vygotski, 1934), où l'Autre est appréhendé en fonction d'un certain mode de réception du sens, lequel est fonction d'une culture enfantine, d'un mode de vie enfantin comme d'un « fond expérientiel» (Colletta, 1995) spécifique à chaque enfant. L'enfant traduit, ce faisant, dans son petit alphabet le grand alphabet de la culture (Lotman, 1990).

Bruner (1991) a constaté notre besoin naturel et précoce de construire narrativement des significations et a présenté le récit comme un des «outils» d'explication de l'extraordinaire comme de l'ordinaire, permettant de rendre compréhensible le comportement d'autrui et plus globalement de donner sens au vécu. A partir de là, nous allons observer la façon dont l'enfant restitue une histoire comme une forme de médiation privilégiée pour observer cette acculturation.

D'un point de vue didactique, la restitution d'un récit est considérée comme une activité interprétative (de la littérature comme du monde) préparatoire à l'entrée dans l'écrit; l'analyse de cette activité permet d'éclairer ce que l'enfant retient, accentue, comprend, et plus globalement interprète d'une lecture et du monde avant même de savoir lire (Cabrejo-Parra E., 2001).

Dans l'activité de restitution, nous considérons que nous n'avons jamais affaire à un texte source et à ses variantes mais toujours à des «reprises-modifications ». Les restitutions sont selon nous toujours des réorganisations, et même des reconstructions du sens par l'enfant.

Les approches considérant une structure sémantique sous-jacente favorisant la mémorisation et donc la restitution du récit (par ex. Kintsch et Van Dijk, 1975, Fayol, 1984) seront aussi prises en compte ; mais nous les modulerons par celles qui soulignent les aspects psycho-sociaux de la mémoire comme étant à l'origine d'une réorganisation dans le récit répété, avec l'introduction de liens non présents dans le texte original, en particulier ceux de causalité (Bartlett, 1932).

Définie comme une conduite discursive liée à une pratique sociale, l'activité de restitution nous semble non seulement liée aux désirs, croyances, attentes propres du sujet mais aussi à la relation à l'interlocuteur et aux circonstances (moment, lieu de restitution).

Cette conception dialogique de la restitution s'inscrit à un niveau plus local dans la lignée des études interactives du récit issues de Labov (1978) qui présentent le genre « récit » comme un genre peu normé, intégrant d'autres genres qui lui sont subordonnés. Labov inclut la présentation d'explications dans l'évaluation, et considère celle-ci comme étant avant tout manifestation de la prise en compte de l'autre en discours, parmi d'autres formes « d'épaississement de la trame narrative » (François, 1993, Préneron \& Salazar-0rvig 1987). 
Cet aspect «dialogique » du genre «secondaire» qu'est l'explication dans un récit a toute sont importance quand il s'agit d'analyser la façon dont les explications ont été restituées : d'un point de vue didactique, savoir restituer des explications incluses dans un récit, c'est d'abord savoir prendre en compte son interlocuteur en lui explicitant, justifiant, expliquant les liens entre les événements. Notons toutefois que l'enchaînement en lui-même des événements peut être explicatif, d'où l'absence de frontières toujours claires entre narration et explication (comme l'a noté Borel 1981 à propos de l'expression « explique-toi »).

L’explication peut cependant être définie plus précisément :

$>$ par le fait de donner du sens par des rapports de causalité, donc comme un processus objectivé,

$>$ par le fait de donner des raisons, mobiles, motifs, mobiles culturels, donc comme une forme de justification, plus psychologique et dialogique. Selon Perelman, la justification présuppose l'éventualité d'une appréciation défavorable concernant ce que l'on s'efforce de justifier, «ne concerne que ce qui est à la fois discutable et discuté » $(1989$, p. 197) : c'est l'écart par rapport à un cadre normatif qui implique une conduite discursive de justification. Ce cadre normatif, proche de l'« idéologie du quotidien » de Bakhtine (1977: 100) ou de «l'opinion du public » de Genette (1969) $)^{1}$ implique des règles et des critères qui diffèrent selon les époques.

On a donc affaire ici à l'arrière-fond que tout récepteur reconstitue. Dans le cas de la restitution d'un récit à l'Ecole, le cadre normatif comporte un double arrière fond :

$>$ celui qui est perceptible dans le TDB,

$>$ celui de l'Ecole, qui propose pour l'activité de restitution de récit certaines normes repérables dans les manuels scolaires et les Normes Officielles, arrière-fond constitué de tout ce qui fait passer le genre textuel récit à un « genre scolaire » (Dolz \& Schneuwly, 1998).

Un autre aspect dialogique des restitutions a été montré très concrètement par Feldman et al. (1990) : le TDB, s'il présente la même trame narrative événementielle et mélange différemment d'autres genres textuels (description, explication, interprétation), génère des types d'interprétations différents en fonction de la dominance de chaque genre.

Très peu de recherches didactiques se sont centrées sur cet aspect spécifique de la restitution, par des enfants, dans le cadre de l'Ecole, d'explications incluses comme ici dans un récit ; cette entrée va nous permettre d'éclairer sous un angle encore peu exploré comment l'enfant se positionne en tant qu'interprète en herbe par rapport à des explications de types différents.

Après avoir présenté la situation de recueil et dégagé 5 types d'explications, ou plutôt de « Mouvements Explicatifs-Justificatifs » (MEJ) dans le Texte de Base (TdB) nous tenterons de répondre à un certain nombre de questions, en particulier :

- Y a-t-il des différences lorsque les enfants restituent l'histoire avec l'aide du livre ou sans ?

- Y a-t-il des enfants qui ne restituent aucun type d'explications d'aucune sorte?

- Y a-t-il un rapport entre le type d'explication restitué et sa place dans la structure TdB ?

- Y a-t-il des explications reprises que de façon partielle?

- Y a t-il des explications, qui relèvent d'un certain type dans le TdB et sont reprises mais dans un autre type dans la restitution?

- Y a-t-il un type d'explication que les enfants restituent plus facilement? 
- Y a-t-il au contraire des types d'explications qui résistent à la mise en mots dans la restitution ? Ou des aspects que nous considérons comme explicatif-justificatif qui ne sont pas repris comme tel par les enfants? Nous verrons en particulier pour cette dernière question que les explications morales et philosophiques, très présentes dans le $\mathrm{TdB}$ disparaissent au profit d'une autre accentuation dans les restitutions des enfants, ce qui aboutit à transformer le genre même du TdB.

Les réponses à l'ensemble de ces questions nous permettront de mieux cerner la façon dont les enfants peuvent avoir accès à un texte littéraire, au niveau de son organisation narrative et surtout de la justification de cette organisation. Ces réponses permettront par là même de cerner quelques spécificités de l'élève-enfant-interprète et donneront des pistes sur la façon de sensibiliser un élève-interprète à la dimension explicative-justificative d'un récit littéraire.

\section{- 2 Présentation du corpus}

Nous allons donc observer ce que deviennent les explications d'un conte dans les restitutions de 3 enfants de GS maternelle. Ces restitutions ont été faites à l'adulte qui avait lu le texte à l'enfant, donc sans enjeu communicatif pour les enfants. Ces trois adultes étaient stagiaires à l'IUFM et se sont isolées dans une salle de l'école, à chaque fois avec un seul enfant, pour lui lire puis lui faire restituer l'histoire, d'abord sans les images de l'album, puis avec afin d'éviter lors de la première restitution toute conduite déictique.

L'album Arc-En-Ciel, le plus beau poisson des océans, de M. Pfister, se présente comme un récit très littéraire au sein duquel les explications, qui appartiennent à des plans différents du récit, contribuent à construire les dimensions morales et philosophiques du conte.

Nous l'avons découpé de façon «classique » (cf. Labov, 1978, Fayol 1984, 1996, Preneron\&Salazar, 1987) en séquences (indication, épisode 1, évaluation, épisode 2, épisode 3, résultat), puis en propositions (cf. annexe 1 pour une présentation du Texte De Base (désormais TDB) et annexe 2 pour une définition de la proposition telle qu'elle a été utilisée ici).

\section{- 3 Les explications dans le récit et leurs restitutions}

\subsection{Cinq types de «mouvements explicatifs-justificatif » dans le TDB}

Certains auteurs ont pointé les différences entre expliquer et justifier en soulignant toutefois un continuum entre les deux (Borel, 1981, Hudelot \&Veneziano, 2005). Pour notre part, nous saisissons le texte dans sa dynamique en pointant des «mouvements» (François, 1993) explicatifs et/ou justificatifs. Nous mettons surtout l'accent sur l'enchaînement des énoncés en contiguïté (micro-mouvement) ou à distance (macro-mouvement). Un mouvement est lié à l'interprétation, au fait de donner sens à l'enchaînement des énoncés. A ce titre, certains mouvements sont fondés sur notre connaissance commune du monde, d'autres sont moins partagés.

Seront considérés ici comme mouvements explicatifs-justificatifs ceux qui sont :

1 relatifs au fait de donner des raisons du dit ou du dire (ou les deux en même temps);

2 postérieurs au fait à expliquer, impliquant une remontée des conséquences vers les causes et donc une perspective de rétrospection (Weinrich, 1973).

Sans ce mouvement, nous considérons qu'il s'agit du déroulement normal du discours qui n'explique pas mais décrit des processus dans leur déroulement causes $\rightarrow$ conséquences, sur un mode proche du script ${ }^{2}$, en présentant une forme d'homologie entre monde réel et monde dit.

Voici les 5 types de Mouvement Explicatif-Justificatif (désormais MEJ) dégagés du TDB :

Tableau 1 


\begin{tabular}{|c|c|c|}
\hline $\begin{array}{c}\text { Types de mouvements } \\
\text { Explicatifs- } \\
\text { Justificatifs (MEJ) }\end{array}$ & Sous-types de MEJ & Exemples \\
\hline \multirow{2}{*}{$\begin{array}{l}\text { MEJ dont le rôle } \\
\text { global et local sont } \\
\text { intriqués. (ces } \\
\text { propositions prennent } \\
\text { une valeur de MEJ } \\
\text { dans le macro- } \\
\text { enchaînement des } \\
\text { paragraphes) } \\
\text { MEJ } 1\end{array}$} & $\begin{array}{l}\text { MEJ de l'articulation } \\
\text { du récit } \\
\text { MEJ } 1.1\end{array}$ & $\begin{array}{l}\text { 29 Mais à quoi servent les plus belles écailles du monde } \\
\mathbf{3 0} \text { s'il n'y a personne pour les admirer? } \\
\text { Question tout à la fois du narrateur et du personnage AEC, qui } \\
\text { fait partie de la morale partagée et qui fait entrer le texte dans } \\
\text { le genre « conte psychologique» }\end{array}$ \\
\hline & $\begin{array}{l}\text { MEJ au niveau des } \\
\text { changements de voix, } \\
\text { de point de vue } \\
\text { MEJ } 1.2\end{array}$ & $\begin{array}{l}33 \text { Un jour il fit part de ses soucis à l'étoile de mer } \\
25 \text { Il raconta aussitôt aux autres poissons sa mésaventure } \\
\text { Souci et mésaventure sont des catégorisations des événements } \\
\text { permettant au narrateur de marquer son point de vue dans le } \\
\text { déroulement du récit. }\end{array}$ \\
\hline $\begin{array}{l}\text { MEJ apparaissant au } \\
\text { cours d'un } \\
\text { changement de plan } \\
\text { énonciatif dans le DR } \\
\text { (point de vue du } \\
\text { personnage) } \\
\text { MEJ } 2\end{array}$ & & $\begin{array}{l}\text { 64 Jamais! Ah non! } \\
\text { 65 Je ne pourrais jamais être heureux sans elles! } \\
<->65 \text { justifie } 64 \text { du point de vue du personnage }\end{array}$ \\
\hline $\begin{array}{l}\text { MEJ dans laquelle } \\
\text { domine l'explicitation } \\
\text { de la psychologie du } \\
\text { personnage à travers } \\
\text { la voix énonciative du } \\
\text { narrateur } \\
\text { MEJ } 3\end{array}$ & & $\begin{array}{l}11 \text { Mais le bel Arc-en ciel glissait près d'eux } \\
12 \text { sans dire un mot, le regard fier } \\
13 \text { en prenant bien soin de faire briller ses écailles. } \\
1^{\circ} \text { niveau : les éléments descriptifs explicitent les formes } \\
\text { prises par l'orgueil et le dédain du personnage. } \\
2^{\circ} \text { niveau : l'explicitation est une forme d'explication des } \\
\text { relations entre les personnages du récit au niveau macro. } \\
<->\text { les éléments de la description dépeignent le personnage } \\
\text { (niveau 1) et rendent la suite possible (niveau 2): }\end{array}$ \\
\hline $\begin{array}{l}\text { MEJ relevant de } \\
\text { l'explication au sens } \\
\text { de la « proposition } \\
\text { explicative » (Labov) } \\
\text { MEJ } 4\end{array}$ & $\begin{array}{l}\text { - marquée } \\
\text { - non marquée }\end{array}$ & $\begin{array}{l}\mathbf{8} \text { Et à cause de ses belles couleurs ils l'avaient nommé Arc-en- } \\
\text { ciel. } \\
\mathbf{8 5} \text { Le petit poisson bleu se promena partout avec sa belle } \\
\text { écaille, } \\
\mathbf{8 6 - e t ~ t o u t ~ l e ~ m o n d e ~ l ' a d m i r a . ~}\end{array}$ \\
\hline $\begin{array}{l}\text { MEJ fonctionnant par } \\
\text { le biais d'évaluations } \\
\text { MEJ } 5\end{array}$ & $\begin{array}{l}\text { lié à la sémantique } \\
\text { et/ou la syntaxe } \\
\text { (adjectif, verbe, } \\
\text { comparaison) }\end{array}$ & 82 Arc-en-ciel se sentit tout drôle \\
\hline
\end{tabular}

Cette typologie en 5 types de MEJ est problématique car on ne peut pas isoler le local du global; dès que l'on « tire « un « fil explicatif», d'autres fils sont aussi à prendre en compte. Les significations sont en effet amalgamées dans le TDB, d'où l'impossibilité d'isoler un type « pur » d'explication dans ce récit.

La proposition 80 " dit le petit poisson tout joyeux » est « classée », à cause de « tout joyeux », comme évaluation (MEJ5) et comme explication de la psychologie du personnage (MEJ2); en même temps, cette proposition est rattachée au fil de la cohérence globale et fait entendre la voix du narrateur qui défend une valeur judéo-chrétienne (MEJ1).

Dans l'exemple ci-dessous (qui reprend de façon parallèle un mouvement déjà tracé précédemment en 9294 avec une justification par le lien donner-être heureux), ce sont les MEJ 4 et 1 qui sont amalgamés :

\section{Extrait TDB 1}

95 Bientôt il ne resta plus à Arc-en-ciel qu'une seule écaille brillante 
96 Il avait distribué toutes les autres !

97 Et il était heureux, vraiment heureux

En fait, l'ensemble des types de MEJ du TDB convergent vers le résultat en tant que « fin » du récit et sont des réaffirmations des mêmes valeurs (générosité, morale du don) contribuant à créer un arrière-fond culturel judéo-chrétien. Les cinq types de MEJ pointés dans le TDB ne nous intéressent finalement pas en tant que typologie, mais par les problèmes qu'elle permet de soulever. En particulier, cette typologie permet d'observer que ce qui, de notre point de vue, est explicatif-justificatif, n'est pas repris comme tel par les enfants. Cette différence nous oblige à réfléchir à la question du genre explicatif, et au fait qu'il est difficilement isolable :

$>$ du TDB car amalgamé à d'autres fils narratifs,

$>$ du narrateur puisque le narrateur-enfant ne produit pas les mêmes explications que le narrateur adulte et n'accentue pas, on va le voir, les mêmes fils explicatifs. 


\subsection{Les types de mouvements explicatifs-justificatifs restitués par les enfants}

Faute de place, nous présentons un tableau récapitulatif dont nous commentons les aspects les plus notoires.

Tableau 2

\begin{tabular}{|c|c|c|c|c|c|c|c|}
\hline $\begin{array}{l}\text { Séquences et } \\
\mathrm{n}^{\circ} \text { des } \\
\text { propositions }\end{array}$ & TDB & $\begin{array}{l}\text { H sans le } \\
\text { livre }\end{array}$ & $\begin{array}{l}\mathrm{H} \text { avec le } \\
\text { livre }\end{array}$ & $\begin{array}{l}\text { C sans le } \\
\text { livre }\end{array}$ & $\begin{array}{l}\mathrm{C} \text { avec le } \\
\text { livre }\end{array}$ & $\begin{array}{l}\text { V sans le } \\
\text { livre }\end{array}$ & $\begin{array}{l}\mathrm{V} \text { avec le } \\
\text { livre }\end{array}$ \\
\hline $\begin{array}{l}\text { Indications } \\
\text { Prop } 1 \text { à } 13\end{array}$ & $\begin{array}{l}\text { EJ5: } \\
3,4,5,6 \\
\text { EJ4 : } 8 \\
\text { EJ3 : } \\
11,12,13\end{array}$ & EJ5 & $\begin{array}{l}\text { EJ4 a } \\
\text { EJ5 } \\
\text { EJ1.2 } \\
\text { EJ4.a }\end{array}$ & EJ5 & $\begin{array}{l}\text { EJ3 } \\
\text { EJ5 }\end{array}$ & EJ5 & EJ5 \\
\hline $\begin{array}{l}\text { Episode } 1 \\
14 \text { à } 28\end{array}$ & $\begin{array}{l}\text { EJ3 : 26- } \\
27-28 \\
\text { EJ2 : 16- } \\
17-22 \\
\text { EJ5.2: } \\
24-25 \\
\end{array}$ & $\begin{array}{l}\text { EJ4 } \\
\text { EJ1.2 } \\
\text { EJ5 }\end{array}$ & $\begin{array}{l}\text { EJ2 } \\
\text { EJ5 }\end{array}$ & & & & EJ3 \\
\hline $\begin{array}{l}\text { Séquence } \\
\text { évaluative } \\
29-32\end{array}$ & $\begin{array}{l}\text { EJ1.2:33 } \\
\text { EJ1.1 : } \\
29-30\end{array}$ & $\begin{array}{l}\text { Séquence } \\
\text { supprimée }\end{array}$ & EJ3 & & & & EJ5 \\
\hline $\begin{array}{l}\text { Episode } 2 \\
33-65\end{array}$ & $\begin{array}{l}\text { EJ4ab : } \\
44-60-51 \\
\text { EJ3 : 33- } \\
34-34-35- \\
36-41-42- \\
55-55-56- \\
65 \\
\text { EJ1 : 55- } \\
56\end{array}$ & $\begin{array}{l}\text { EJ2 } \\
\text { EJ3 } \\
\text { EJ5 }\end{array}$ & $\begin{array}{l}\text { EJ3 } \\
\text { EJ4.b }\end{array}$ & $\begin{array}{l}\begin{array}{l}\text { EJ5 } \\
\text { (mais) }\end{array} \\
\text { EJ4.a } \\
\text { EJ1 } \\
\text { EJ2 }\end{array}$ & $\begin{array}{l}\begin{array}{l}\text { EJ5 } \\
\text { (mais) }\end{array} \\
\text { EJ4.a } \\
\text { EJ1 } \\
\text { EJ2 }\end{array}$ & & $\begin{array}{l}\text { EJ3 } \\
\text { EJ4a } \\
\text { EJ2 }\end{array}$ \\
\hline $\begin{array}{l}\text { Episode } 3 \\
66-94\end{array}$ & $\begin{array}{l}\text { EJ4 : 67- } \\
85-88 \\
\text { EJ3 : 80- } \\
82-89-94 \\
\text { EJ2 : } 74 \\
\text { EJ1.2 : } \\
74-80-82- \\
93-94 \\
\end{array}$ & $\begin{array}{l}\text { EJ4 } \\
\text { EJ2 }\end{array}$ & & & & & $\begin{array}{l}\text { EJ2 } \\
\text { EJ5 }\end{array}$ \\
\hline $\begin{array}{l}\text { Résultat } \\
95-102\end{array}$ & $\begin{array}{l}\text { EJ4 : } 96 \\
\text { EJ1.2 : } \\
95-97 \\
\end{array}$ & & & & & & \\
\hline
\end{tabular}

Ce tableau nous permet d'observer un certain nombre de conduites discursives chez les enfants qui demanderaient à être confirmées et discutées sur des données plus vastes :

$>$ Le livre aide à la restitution des MEJ.

$>$ Tous reprennent au moins une fois chaque type de MEJ, sauf $\mathrm{V}$ qui ne reraconte pas sans le livre, et qui est la seule à ne pas avoir de MEJ 1 (ni avec ni sans le livre).

$>$ Il y a une affinité entre le type de MEJ et la structure du TDB : les MEJ de la séquence indications, en particulier les évaluations (MEJ5), sont repris chez tous avec ou sans le livre, même si c'est parfois de façon partielle.

V. sans le livre utilise une comparaison et un superlatif (soulignés) : 


\section{Exemple 1}

il était une + fois, un poisson $<$ une inspiration $>$ hum coloré comme un arc-en-ciel $(\ldots)$

Il é-tait le plus beau des poissons de la $<$ baissant la voix $>$ mer.

> les MEJ de l'épisode 1 ne sont repris que par $\mathrm{H}$ avec et sans le livre, et par $\mathrm{V}$ avec le livre,

> les MEJ de la séquence évaluation sont repris par $\mathrm{H}$ et $\mathrm{V}$ avec le livre,

$>$ les MEJ de l'épisode 2 sont repris par tous sauf V sans livre,

$>$ les MEJ de l'épisode 3 sont repris par H sans le livre et par V avec le livre,

$>$ les MEJ du résultat ne sont repris par personne.

Parfois les MEJ ne sont pas à proprement reprises mais transformées, et passent d'un type à un autre. Par exemple, $\mathrm{C}$ avec le livre reprend seulement un élément, certes essentiel, de la séquence indication, à savoir la relation entre la couleur d'AEC et l'admiration des autres :

\section{Exemple 2}

$<$ tourne la page $>$ Alors + bon + tout au fond de l'o-cé-an vivait un petit poisson-ON

de toutes les couleurs. Ah et tous les autres poissons l'admiraient

en reprenant-modifiant les propositions 1, 4, $7 \mathrm{du}$ TDB :

\section{Extrait TDB 2}

1 Quelque part au plus profond des mers vivait un poisson

4 Il avait les couleurs de l'arc-en-ciel,

7 Les autres poissons admiraient ses écailles scintillantes...

L'auteur du TDB focalise d'abord sur la description du personnage (propositions 3 à 5 , relevant d'un MEJ 5) pour ensuite pointer la relation entre les autres poissons et AEC (proposition 8, relevant d'un MEJ 4), et celle d'AEC avec les autres poissons (propositions 11 à 13, relevant d'un MEJ 3).

$\mathrm{C}$ reprend les évaluations de la proposition 4 (les couleurs) ainsi que la proposition descriptive 7 du TDB, mais en lui donnant une valeur explicative, opérant ce faisant une condensation du TDB et actualisant du même coup un MEJ 3 (voix énonciative du narrateur) différent de celui du TDB.

Par ailleurs, C sans le livre reprend un MEJ de l'épisode 2 du TDB (proposition 60) :

\section{Extrait TDB 3}

59 Il ne put rien ajouter de plus

60 car Octopus avait déjà disparu derrière un nuage d'encre.

Mais alors que la proposition 60 du TDB était un MEJ 4 relevant du plan de l'énonciation, C le transforme dans sa restitution en scène relevant de l'énoncé (MEJ 5) (souligné dans l'exemple qui suit) : 


\title{
Exemple 3
}

\begin{abstract}
(...) la pieuvre elle lui a dit «do-donne à chaque poisson qui te demanderait des écAILLES :de tes écailles brillantes $+<$ raclement de gorge $>$ Tu leu :r donne'ras une des tes écailles Brillantes $<$ pause $5 \mathrm{sec}><$ raclement de gorge $>$ Et, et Apres elle disait $<$ plus fort en changeant de voix $>$ « une de mes écailles briLLAN ::TES » dit Arc-enCiel. Mais Octopu-us la pieuvre avait déjà-a disparu.
\end{abstract}

Les conseils de la pieuvre sont repris par $\mathrm{C}$ sans qu'il y ait problématisation, même s'il y a quand même un MEJ 2 (changement de plan énonciatif), un MEJ 4 « discours rapporté » et un MEJ 5 (« mais ») : le personnage d'AEC ne formule plus sa question et est confronté à une parole d'autorité, donnée dans une forme d'opacité, telle la parole de l'oracle, sans la justification du conseil présente dans le TDB, relevant d'un MEJ2, et qui faisait appel à la raison :

\section{Extrait TDB 4}

55 Tu ne seras peut-être plus le plus beau des poissons

56 mais tu seras un poisson heureux

De ce fait, il y a un passage d'un monde rationnel à un monde magique, et donc une autre tension que celle du TDB, avec la présence d'un MEJ 1 (intrication du sens local et global mais qui prend un autre sens que celui de TDB).

Il y a une tendance chez tous à donner des explications par le biais des évaluations (MEJ 5), mais d'une façon très différente du TDB :

$>\quad$ intonatifs chez V. (cf. dernier exemple);

$>$ sans lien avec l'explication chez C. dont le récit est pure succession d'événements sans dimension psychologique (suite de l'exemple précédent) :

\section{Exemple 4}

(...)Et le petit poisson bleu lui dit» S'il te plaît Arc-en-ciel donne moi une de tes écailles ! »+Et il dit + et il dit « OUI !Alors une toutes petite+ D’ACCORD!» $<$ tourne la page> Il détache une de ses écailles brillantes + et i dit «MERCI!» $<$ tourne la page $>$ Après tous les poissons en voulaient + une. Et après il distri-buait :: $<$ tourne la page $>$ Arc-en-ciel toutes ses écailles. Il en avait plus qu'UNE $<$ tourne la page $>$ Tous les autres en avaient $<$ tourne la page, inspiration $>$ Et+et voilà !

Cette restitution comporte une tension minimale entre le début et la fin, sans laquelle on aurait pu se demander si on pouvait encore la qualifier de « récit».

Les MEJ 3 sont très peu repris, les MEJ 1 encore moins, tandis que l'on note une extension de MEJ 2 : tout se passe comme si les MEJ 5 et les MEJ 2 prenaient la place des MEJ 3 et des MEJ 1. Ce phénomène s'explique en partie par le fait que les enfants ne «prennent» pas la place de commentaire du narrateur mais plutôt la place du héros vivant une aventure, sans affirmer à partir des événements des valeurs moralo-philosophiques. Ainsi, la voix du narrateur (transmise par les MEJ de type 1) «bascule» dans celle des personnages (MEJ2) sous une forme affaiblie : le comportement des personnages est expliquéjustifié par une relation cause-conséquence plus événementielle que psychologique. Par exemple chez $\mathrm{H}$. il s'agit d'une psychologie opposant content/pas content mais il n'y a plus la projection d'un regard tiers.

L'extrait suivant (restitution sans le livre) reprend l'épisode 3 mais en faisant disparaître toutes les formes de MEJ :

\section{Exemple 5}

(...) et la pieuvre elle a dit «si tu veux être content, il faut donner une écaille à tes copains »

Plus localement, elle reprend une partie de l'épisode 1 


\section{Extrait TDB 5}

26 effrayé, le petit poisson bleu s'enfuit d'un vif coup de nageoire

27 il raconta aussitôt aux autres poissons sa mésaventure.

et supprime dans la reprise de ces deux propositions la voix du narrateur (le MEJ1.2) ; son explication devient purement causale (MEJ4) :

\section{Exemple 6}

$$
\text { « (...) alors il est reparti, le poisson bleu ». }
$$

Dans sa restitution avec le livre, le comportement du poisson est repris sous forme d'évènement :

\section{Exemple 7}

$$
(\ldots)<\text { tourne la page> Et euh et pis y a tout le monde il dit « tu tu viens on va jouer ? » }
$$
Mais non non lui il est reparti sans dire un mot (...)

Dans la restitution de V (avec le livre), qui est la plus proche du TDB, la dimension morale disparaît aussi même s'il y a une forme de reprise partielle dans du discours rapporté (et non par le commentaire d'un narrateur extérieur au récit) :

\section{Exemple 8}

(...) Arc-en-ciel pens:SA + «donner une : + de mes écailles brillantes+oh n-ça NON! je s'rai + jamais heureux sans elles!»<tourne la page> ++ hum++hum++hum :+le petit poisson bleu arriva. "S'il te plait Arc-en-ciel donne-moi une de tes écailles sois gentil+elles sont si br-elles »+D'ACCORD une petite » pensa Arc-en-ciel <tourne la page > Arc-en-ciel détache ;+délicatement UNE de ses écailles la plus petite $(\mathrm{X})+$ «Allez maintenant va-t-en! »+»Oh oui merci+MERCI!» $<$ tourne la page>le petit poisson bleu euh : nagea long ::temps et $<$ soupir $>$ à un moment, Arc-en-ciel fut Entouré de petits poissons. Ils voulaient chacun thum une écaille : <tourne la page $>»$ Viens jouer avec nous maintenant Arc-en-ciel! Oh OUI! Viens jouer avec nous! »Et voilà.

C'est ici le contraste de situation entre «Viens jouer avec nous » et le fait qu'Arc-en-ciel était présenté au début comme seul

\section{Exemple 9}

Arc-en-ciel était + non pas le plus beau des poissons, mais aussi le plus SEUL des poissons

qui restitue la morale de l'histoire sous une forme très affaiblie, non explicite.

\section{- 4 Conclusion}

Les enfants sont sensibles aux évaluations locales et descriptives du personnage (MEJ 5), non à la dimension morale et philosophique du conte, ni à la voix du narrateur qui porte cette dimension. Ils perçoivent la façon de se comporter du héros comme un enchaînement de causes et d'effets et sont sensibles aux nœuds narratifs de l'ordre du psychologique dans la mesure où ils forment des motifs du type « ne pas être gentil/être puni », « ne pas prêter/être tout seul ».

Les enfants ne «prennent » pas la place du narrateur du TDB formulant des commentaires distanciés du mais davantage une place de héros vivant une aventure ; ce qui est en affinité avec le mode de narration sous forme de nœuds événementialo-psychologiques.

La dimension morale ressort de ce fait sous une forme très affaiblie, voire absente pour certains : les enfants n'étant plus dans le même système de valeur ni à la même place narrative que le narrateur du TDB. De ce fait, le sous-genre «conte moral» du TDB est repris-modifié pour devenir «conte événementiel ». 
Ce changement d'accentuation nous permettrait d'avoir accès à une forme de culture enfantine. L'activité de restitution, mais aussi la relative «malléabilité » de la forme du récit, permettent ainsi une forme de médiation entre culture enfantine et culture adulte.

D'un point de vue didactique, cette étude apporte un éclairage sur la façon de circonscrire une zone proximale de développement dans laquelle l'adulte peut se placer quand il aide un enfant à comprendre et interpréter un texte littéraire : par exemple, les aspects les moins restitués, ici les aspects philosophiques et moraux pourraient être retenus pour servir de base à un débat interprétatif permettant aux enfants de construire du sens grâce au guidage de l'enseignant.

\section{- Bibliographie}

Bakhtine M., 1977, Le marxisme et la philosophie du langage, (essai d'application de la méthode sociologique en linguistique), Paris, Éditions de Minuit.

Bartlett F.C., 1932, Remembering: a study in experimental and social psychology, Cambridge, Cambridge University Press.

Borel M.J., 1981, "L'explication dans l'argumentation, approche sémiologique" in Langue française $n^{\circ}$ 50 : argumentation et énonciation, pp. 20-38.

Cabrejo-Parra E., 2001, « La lecture avant les textes écrits » in Cahiers A.C.C.E.S, n5, pp. 19-23.

Colletta J.M., 1995, «Qui parle et pourquoi ? Examen critique de quelques approches de l'interaction », in Lidil $n^{\circ} 12$ : l'interaction en questions, pp. 43-67.

Bruner J., 1991 (ed. or. 1990), Car la culture donne forme à l'esprit, Paris : Eshel.

Dolz, J. \& Schneuwly B., 1998, Pour un enseignement de l'oral. Initiation aux genres formels à l'école, ESF Editeurs, Paris.

Fayol M., 1984, Le récit et sa construction, Neuchâtel-Paris, Delachaux et Niestlé.

Fayol M., 1996, «La production d'écrits narratifs: approche de psycholonguistique textuelle chez l'enfant et l'adulte » in J. David et S. Plane (Eds.) (1996), L'apprentissage de l'écriture de l'école au collège, Paris, Presses Universitaires de France, pp. 9-36.

Feldman C. F., Bruner J., Renderer B., Spitzer S., "Narrative Comprehension", in Britton B. K., Pellegrini A.D., 1990, Narrative Thought and Narrative Language, Lawrence Erlbaum Associates, New Jersey, 278 p, pp. 1-54.

François F., 1993, Pratiques de l'oral, Paris, Nathan.

Genette G., 1969, «Vraisemblance et motivation » in Figures 2, Paris, Seuil.

Labov W., 1978, « La transformation du vécu à travers la syntaxe narrative », in Le parler ordinaire, Paris, Editions de Minuit, pp. 289-335.

Lotman Y., M., 1990, Universe of the mind : a semiotic theory of culture, Bloomington : Indiana University Press.

Perelman C., 1989, «Jugements de valeur, justification et argumentation » in Rhétoriques, Ed. de l'Université de Bruxelles, Bruxelles, 1989, pp. 197-207. (Première parution : 1961.)

Kintsch W., Van Dijk T., 1975, « Comment on se rappelle et on résume des histoires » in Langage n 40, pp. 98-125.

Preneron C., Salazar A., 1987, «La conduite de récit chez des enfants non-lecteurs » in La psychanalyse de l'enfant, vol. 3-4, pp. 44-94.

Propp V., 1928, Morphologie du conte, Paris, Seuil.

Veneziano E. \& Hudelot C., 2005, «Conduites explicatives dans la narration et effet de l'étayage : Méthodes d'analyse et quelques résultats qualitatifs tirés d'une étude développementale et comparative d'enfants typiques et d'enfants dysphasiques », TRANEL (Travaux neuchâtelois de linguistique), 42, 81-103

Vygotski L. S., 1997 (éd. or. 1934), Pensée et langage, Paris, La Dispute.

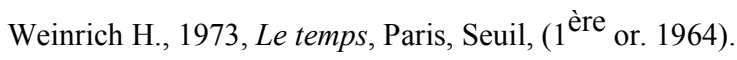




\section{ANNEXE 1: Découpage du TDB en séquences (les retours à la l. correspondent à une nouvelle p. du livre). \\ INDICATIONS}

Quelque part au plus profond des mers vivait un poisson. Mais ce n'était pas un poisson ordinaire : c'était le plus beau poisson de tous les océans. Il avait les couleurs de l'arc-en-ciel, et ses écailles brillaient et miroitaient dans la lumière comme autant de petites gouttes irisées.

Les autres poissons admiraient ses écailles scintillantes...Et à cause de ses belles couleurs ils l'avaient nommé Arc-enciel. « Arc-en-ciel, viens jouer avec nous ! » disaient-ils. Mais le bel Arc-en-ciel glissait près d'eux sans dire un mot, le regard fier, en prenant bien soin de faire-briller ses écailles

\section{EPISODE 1 : le poisson bleu}

Un petit poisson bleu le suivit. «Hé ! Arc-en-ciel, attends-moi! Donne-moi une de tes écailles, elles sont si belles, et tu en as tellement!

« Te donner une de mes écailles? Mais qu'est-ce que tu imagines! Allons, ouste! Déguerpis! » S'exclama Arc-en-ciel avec dédain. Effrayé, le petit poisson bleu s'enfuit d'un vif coup de nageoire. Il raconta aussitôt aux autres poissons sa mésaventure. A partir de ce jour là plus personne ne voulut adresser la parole à Arc-en-ciel. Et quand il passait près d'eux les autres poissons se détournaient

\section{EVALUATION}

Mais à quoi servent les plus belles écailles du monde s'il n'y a personne pour les admirer? A présent Arc-en-ciel n'était plus seulement le plus beau poisson des océans c'était aussi le plus seul.

\section{EPISODE 2 : la pieuvre}

Un jour il fit part de ses soucis à l'étoile de mer. «Je suis si beau, lui dit-il, pourquoi est-ce qu'on ne m'aime pas?» L'étoile de mer se tut un instant puis elle dit : « Derrière le récif de corail il y a une grotte, c'est là qu'habite Octopus, la pieuvre. Elle sait beaucoup de choses, peut-être pourra t-elle te donner un conseil. »Arc-en-ciel ne tarda pas à trouver la grotte. Comme il y faisait sombre! On n'y voyait presque rien. Soudain il vit briller deux yeux dans l'obscurité. Trois bras s'avancèrent vers lui.

« Je t'attendais, dit Octopus d'une voix grave, les vagues m'ont rapporté ton histoire. Ecoute bien mon conseil : offre à chaque poisson qui te le demandera l'une de tes belles écailles. Tu ne seras peut-être plus le plus beau des poissons, mais tu seras un poisson heureux. »

«Mais » dit Arc-en-ciel. Il ne put rien ajouter de plus car Octopus avait déjà disparu derrière un nuage d'encre. Offrir mes écailles? Mes magnifiques écailles? Pensa t-il indigné. Jamais! Ah non! Je ne pourrais jamais être heureux sans elles!

\section{EPISODE 3 : distribution des écailles}

Tout à coup il sentit l'eau vibrer. Le petit poisson bleu était à nouveau près de lui. « Arc-en-ciel, ....donne-moi une de tes magnifiques écailles! » s'il te plaît, sois gentil,Arc-en-ciel hésita. Une toute petite écaille, pensa-t-il, bon, d'accord, ça ne se verra pas.

Arc-en-ciel détacha avec précaution la plus petite écaille de son dos et la donna au poisson bleu. « Tiens, la voilà! Et maintenant va-t-en ! » « Oh ! Merci, merci beaucoup! dit le petit poisson tout joyeux, c'est vraiment très gentil de ta part, Arc-en-ciel! » Arc-en-ciel se sentit tout drôle. Il regarda longuement le petit poisson bleu s'éloigner avec sa petite écaille scintillante.

Le petit poisson bleu se promena partout avec sa belle écaille, et tout le monde l'admira. Bientôt Arc-en-ciel fut entouré d'une nuée de petits poissons : ils voulaient tous une écaille !Et, qui l'eut cru, Arc-en-ciel se mit à distribuer ses belles écailles. Cela l'amusait même de voir la joie des petits poissons. Et plus il voyait scintiller de poissons autour de lui, mieux il se sentait au milieu d'eux

\section{RESULTAT}

Bientôt il ne resta plus à Arc-en-ciel qu'une seule écaille brillante. Il avait distribué toutes les autres! Et il était heureux, vraiment heureux. "Viens jouer avec nous, Arc-en-ciel! » appelèrent les autres poissons. «J'arrive ! » dit Arc-en-ciel, et il s'élança tout joyeux au milieu de ses nouveaux camarades 


\section{ANNEXE 2 : définition du terme proposition}

Les propositions ont été identifiées selon un critère syntaxique afin de travailler avec un seul prédicat (donc, avec, le plus souvent, une seule action) son sujet et ses expansions et cerner ainsi au plus près les conduites de reprise et de reformulation des enfants. Ces propositions ne coïncident pas nécessairement avec les propositions narratives. Elles se présentent de trois façons :

- Elles correspondent à une proposition narrative identifiée par C. Brémond (Brémond, 1973), comme un processus-prédicat et un personnage sujet (agent ou patient).

\begin{tabular}{|l|l|l|}
\hline Saynète & $N^{\circ}$ de proposition & Propositions du récit d'origine \\
\hline S4 & P 033 & Un jour il fit part de ses soucis à l'étoile de mer. \\
\hline
\end{tabular}

La proposition $\mathrm{P} 033$ correspond à une proposition narrative.

- Elles correspondent à des composantes de propositions narratives. Il peut s'agir de propositions coordonnées ou subordonnées du point de vue syntaxique dans une même phrase :

\begin{tabular}{|l|l|l|}
\hline Saynètes & $\mathrm{N}^{\circ}$ de proposition & Propositions du récit d'origine \\
\hline S5 & P 059 & Il ne put rien ajouter de plus \\
\hline S5 & P 060 & car Octopus avait déjà disparu derrière un nuage d'encre \\
\hline
\end{tabular}

Ici les deux propositions sont considérées comme articulées par la conjonction de coordination « car ».

- Enfin, deux propositions peuvent constituer une seule unité narrative.

\begin{tabular}{|l|l|l|}
\hline Saynètes & $\mathrm{N}^{\circ}$ de proposition & Propositions du récit d'origine \\
\hline S1 & P 009 & « Arc-en-ciel, viens jouer avec nous ! » \\
\hline S1 & P 010 & disaient-ils \\
\hline
\end{tabular}

L'attribution du discours rapporté fonctionne en lien avec la proposition P 009. C'est dans ce sens que ces deux propositions forment une unité narrative dans la saynète 1 .

Sur le plan narratif, les propositions peuvent jouer plusieurs rôles. Elles peuvent être équivalentes à des propositions centrales, faire partie de la dramatisation, coder des événements...

1 c'est-à-dire « un corps de maximes et de préjugés qui constitue tout à la fois une vision du monde et un système de valeurs » $(1969$, p. 73).

2 L'ordre des propositions dans un récit induit des relations de causalité de type explicatif: un certain nombre de faits s'enchaînent naturellement, ce qui vient après semblant découler de ce qui précède : c'est cette perspective qui a été d'ailleurs privilégié par les héritiers de Propp (1928) dans une analyse " logique ", " chronologique » du récit. 